\title{
Abundant Z-cyanomethanimine in the interstellar medium: paving the way to the synthesis of adenine
}

\author{
V. M. Rivilla ${ }^{1 \star}$, J. Martín-Pintado², I. Jiménez-Serra², S. Zeng ${ }^{3}$, S. Martín ${ }^{4,5}$, \\ J. Armijos-Abendaño ${ }^{6}$, M. A. Requena-Torres ${ }^{7}$, R. Aladro ${ }^{8}$, and D. Riquelme ${ }^{8}$ \\ ${ }^{1}$ INAF-Osservatorio Astrofisico di Arcetri, Largo Enrico Fermi 5, I-50125, Florence, Italy \\ ${ }^{2}$ Centro de Astrobiología (CSIC-INTA). Ctra de Ajalvir, km. 4, Torrejón de Ardoz, 28850 Madrid, Spain \\ ${ }^{3}$ School of Physics and Astronomy, Queen Mary University of London, Mile End Road, E1 4NS London, United Kingdom \\ ${ }^{4}$ European Southern Observatory (ESO), Alonso de Córdova 3107, Vitacura, Santiago, Chile \\ ${ }^{5}$ Joint ALMA Observatory, Alonso de Córdova 3107, Vitacura, Santiago, Chile \\ ${ }^{6}$ Observatorio Astronómico de Quito, Escuela Politécnica Nacional, Av. Gran Colombia S/N, Interior del Parque La Alameda, \\ 170136, Quito, Ecuador \\ ${ }^{7}$ University of Maryland, College Park, ND 20742-2421 \\ ${ }^{8}$ Max-Planck-Institut für Radioastronomie, Auf dem Hügel 69, 53121 Bonn,
}

Accepted XXX. Received YYY; in original form ZZZ

\begin{abstract}
We report the first detection in the interstellar medium of the Z-isomer of cyanomethanimine (HNCHCN), an HCN dimer proposed as precursor of adenine. We identified six transitions of Z-cyanomethanimine, along with five transitions of Ecyanomethanimine, using IRAM 30m observations towards the Galactic Center quiescent molecular cloud $\mathrm{G}+0.693$. The Z-isomer has a column density of $(2.0 \pm 0.6) \times 10^{14}$ $\mathrm{cm}^{-2}$ and an abundance of $1.5 \times 10^{-9}$. The relative abundance ratio between the isomers is $[\mathrm{Z} / \mathrm{E}] \sim 6$. This value cannot be explained by the two chemical formation routes previously proposed (gas-phase and grain surface), which predicts abundances ratios between 0.9 and 1.5. The observed $[\mathrm{Z} / \mathrm{E}]$ ratio is in good agreement with thermodynamic equilibrium at the gas kinetic temperature (130-210 K). Since isomerization is not possible in the ISM, the two species may be formed at high temperature. New chemical models, including surface chemistry on dust grains and gas-phase reactions, should be explored to explain our findings. Whatever the formation mechanism, the high abundance of Z-HNCHCN shows that precursors of adenine are efficiently formed in the ISM.
\end{abstract}

Key words: Galaxy: centre - ISM: molecules - ISM: abundances -ISM: clouds

\section{INTRODUCTION}

Understanding the origin of life on Earth is one of the most challenging problems in astrophysics in the framework of astrobiology. To shed light on this complex topic, it is absolutely needed a comprehensive study of the chemical complexity of the interstellar medium (ISM) that feeds the formation of stars and planets. In this sense, the chemical family of nitriles can give important clues. Nitriles, organic compounds with a $-\mathrm{C} \equiv \mathrm{N}$ functional group, play a crucial role in prebiotic chemistry since they are key intermediates in the formation of amino acids, peptides, nucleic acids and nucleobases (e.g. Balucani 2009). Adenine $\left(\mathrm{H}_{5} \mathrm{C}_{5} \mathrm{~N}_{5}\right)$, one of the nucleobases of DNA and RNA, is a basic ingredient in the RNA-world scenario for the origin of life

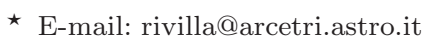

on Earth (e.g. Kaiser \& Balucani 2001, Ehrenfreund et al. 2001, Bernstein et al. 2004). Oró (1961) reported the synthesis of adenine from a solution of $\mathrm{HCN}$ and $\mathrm{NH}_{3}$ under conditions similar to those thought to have existed on the primitive Earth. Chakrabarti \& Chakrabarti (2000) proposed that adenine might be formed during the chemical evolution of a star-forming molecular cloud through the oligomerization of $\mathrm{HCN}$ in the gas phase in four steps:

$$
\begin{aligned}
& \mathrm{HCN} \rightarrow \mathrm{H}_{2} \mathrm{C}_{2} \mathrm{~N}_{2} \rightarrow \mathrm{NH}_{2} \mathrm{CH}(\mathrm{CN})_{2} \rightarrow \\
& \rightarrow \mathrm{NH}_{2}(\mathrm{CN}) \mathrm{C}=\mathrm{C}(\mathrm{CN}) \mathrm{NH}_{2} \rightarrow \mathrm{H}_{5} \mathrm{C}_{5} \mathrm{~N}_{5}
\end{aligned}
$$

However, by performing theoretical calculations, Smith et al. (2001) and Yim \& Choe (2012) showed that the first step, i.e. the formation of an HCN dimer from two HCN molecules, does not occur efficiently in the conditions of the 
ISM. Therefore, the question of how HCN dimers can be formed remains open. Since they are precursors of adenine, understanding their formation mechanisms is of crucial importance from an astrobiological point of view.

The most stable dimer of $\mathrm{HCN}$ is C-cyanomethanimine (HNCHCN hereafter), which presents two different isomers: the Z-isomer and the E-isomer (Clemmons et al. 1983). These species are stereoisomers about the double bond $\mathrm{N}=\mathrm{C}$ (see e.g. Fig. 1 of Takano et al. 1990) and the conversion from the Z- to the E-isomer requires an energy of $15.95 \mathrm{kK}$. The laboratory experiments and chemical calculations by Takano et al. (1990) and Zaleski et al. (2013), respectively, indicate that the Z-isomer is more stable and lower in energy than the E-isomer, with an estimated energy difference in the range 238-382 K. Nevertheless, only the highenergy E-isomer has been detected in the ISM so far. Several lines were reported in absorption towards the bright continuum of the cluster of hot cores located in the SgrB2N complex (Zaleski et al. 2013), while the Z-conformer was elusive. Recent searches of the Z-conformer in a sample of low-mass star-forming regions have also been unsuccessful (Melosso et al. 2018).

In this work, we report the results of an interstellar search for the Z-conformer of $\mathrm{HNCHCN} \mathrm{(Z-}$ HNCHCN hereafter). Using an IRAM $30 \mathrm{~m}$ spectral survey, we searched for this species in the $G+0.693-0.027$ giant molecular cloud $(\mathrm{G}+0.693$ hereafter) in the Galactic Center. This region exhibits high gas kinetic temperatures ranging from $\sim 50 \mathrm{~K}$ to $\sim 150 \mathrm{~K}$ (Guesten et al. 1985; Hüettemeister et al. 1993; Rodríguez-Fernández et al. 2001; Ginsburg et al. 2016; Krieger et al. 2017; Zeng et al. 2018), low dust temperatures of $\leq 30 \mathrm{~K}$ (Rodríguez-Fernández et al. 2004), and relatively low $\mathrm{H}_{2}$ gas densities $\left(\sim 10^{4} \mathrm{~cm}^{-3}\right.$; Rodríguez-Fernández et al. 2000). Due to the low $\mathrm{H}_{2}$ densities, the molecules are sub-thermally excited and hence the excitation temperatures are low $(\sim 5-20 \mathrm{~K}$; Requena-Torres et al. 2006; Martín et al. 2008; Rivilla et al. 2018; Zeng et al. 2018). G+0.693 is one of the most chemically rich reservoirs in our Galaxy. Many molecular species have been identified in this cloud, including some of prebiotic relevance such as complex organic molecules (COMs; Requena-Torres et al. 2006, 2008; Zeng et al. 2018) and phosphorus-bearing species (Rivilla et al. 2018). In particular, numerous nitrile molecules have been already detected in the source such as $\mathrm{C}_{3} \mathrm{~N}, \mathrm{HC}_{3} \mathrm{~N}, \mathrm{HC}_{5} \mathrm{~N}, \mathrm{HC}_{7} \mathrm{~N}, \mathrm{CH}_{2} \mathrm{CN}$, $\mathrm{CH}_{3} \mathrm{CN}, \mathrm{CH}_{3} \mathrm{C}_{3} \mathrm{~N}, \mathrm{NH}_{2} \mathrm{CN}$ and $\mathrm{HOCN}$ (Zeng et al. 2018). This rich nitrile chemistry makes this source an excellent target to search for more complex nitriles, and in particular Z-HNCHCN.

\section{OBSERVATIONS}

We used in this work a spectral line survey towards the quiescent molecular cloud $\mathrm{G}+0.693$ carried out with the IRAM
Table 1. Transitions of HNCHCN detected towards G+0.693.

\begin{tabular}{|c|c|c|c|c|c|}
\hline $\begin{array}{c}\text { Frequency } \\
\quad(\mathrm{GHz})\end{array}$ & Transition & $\begin{array}{c}\log \mathrm{A}_{\mathrm{ul}} \\
\left(\mathrm{s}^{-1}\right)\end{array}$ & $\begin{array}{l}\mathrm{E}_{\text {up }} \\
(\mathrm{K})\end{array}$ & $\underset{\left(\mathrm{K} \mathrm{km} \mathrm{s}^{-1}\right)}{T_{\mathrm{A}}^{*} d \mathrm{v}}$ & $\begin{array}{l}\text { Detection } \\
\text { level }\end{array}$ \\
\hline \multicolumn{6}{|c|}{ Z-isomer } \\
\hline 85.283 & $9(1,9)-8(1,8)$ & -5.2107 & 23 & $0.68 \pm 0.25$ & 13.8 \\
\hline 86.996 & $9(0,9)-8(0,8)$ & -5.1795 & 21 & $0.91 \pm 0.30$ & 15.9 \\
\hline 89.247 & $9(1,8)-8(1,7)$ & -5.1515 & 24 & $0.63 \pm 0.24$ & 7.7 \\
\hline 94.735 & $10(1,10)-9(1,9)$ & -5.0705 & 27 & $0.47 \pm 0.21$ & 13.7 \\
\hline 96.569 & $10(0,10)-9(0,9)$ & -5.0413 & 26 & $0.62 \pm 0.25$ & 7.1 \\
\hline 109.017 & $11(1,10)-10(1,9)$ & -4.8849 & 34 & $0.27 \pm 0.16$ & 4.8 \\
\hline \multicolumn{6}{|c|}{ E-isomer } \\
\hline 84.425 & $9(1,9)-8(1,8)$ & -4.4608 & 23 & $0.71 \pm 0.05$ & 13.9 \\
\hline 93.791 & $10(1,10)-9(1,9)$ & -4.3204 & 28 & $0.51 \pm 0.04$ & 11.3 \\
\hline 95.422 & $10(0,10)-9(0,9)$ & -4.2937 & 25 & $0.71 \pm 0.05$ & 17.9 \\
\hline 97.501 & $10(1,9)-9(1,8)$ & -4.2698 & 29 & $0.47 \pm 0.04$ & 8.4 \\
\hline 104.895 & $11(0,11)-10(0,10)$ & -4.1684 & 30 & $0.46 \pm 0.03$ & 6.9 \\
\hline
\end{tabular}

$30 \mathrm{~m}$ telescope at Pico Veleta ${ }^{1}$ (Spain) and the $\mathrm{NRAO}^{2} 100 \mathrm{~m}$ Robert C. Byrd Green Bank telescope (GBT) in West Virginia (USA), covering frequencies from 12 to $272 \mathrm{GHz}$. The observations were centered at the coordinates $\alpha(\mathrm{J} 2000.0)=$ $17^{h} 47^{m} 22^{s}$ and $\delta(\mathrm{J} 2000.0)=-28^{\circ} 21^{\prime} 27^{\prime \prime}$. We refer to Zeng et al. (2018) for more detailed information on the observations.

\section{ANALYSIS AND RESULTS}

The identification of the lines was performed using the SLIM (Spectral Line Identification and Modeling) tool of the MADCUBA package ${ }^{3}$, which contains the spectroscopic information from the Cologne Database for Molecular Spectroscopy $\left(\mathrm{CDMS}^{4}\right)$ (Müller et al. 2001, 2005; Endres et al. 2016) and the Jet Propulsion Laboratory (JPL) catalogue ${ }^{5}$ (Pickett et al. 1998). We have used the CDMS entries for the two isomers of HNCHCN, which contain the spectrocopy from Takano et al. (1990), Zaleski et al. (2013) and Melosso et al. (2018). Since there are not collisional coefficients available for these species we performed the analysis assuming local thermodynamic equilibrium conditions (LTE). We generated with SLIM-MADCUBA a synthetic spectrum to compare with the observations. We confirmed the presence of ten transitions of Z-HNCHCN in the spectra towards $\mathrm{G}+0.693$ with a significant detection level $(>4 \sigma$; see below). Six of these transitions are unblended, i.e., they are not contaminated by emission from other molecular

1 This paper makes use of observations obtained with the IRAM$30 \mathrm{~m}$ telescope. IRAM is supported by INSU/CNRS (France), MPG (Germany), and IGN (Spain).

2 The National Radio Astronomy Observatories is a facility of the National Science Foundation, operated under a cooperative agreement by Associated Universities, Inc.

3 Madrid Data Cube Analysis on ImageJ is a software developed at the Center of Astrobiology (CAB) in Madrid; http://cab.inta-csic.es/madcuba/Portada.html; see Martín et al., in prep; Rivilla et al. (2016, 2017a).

${ }^{4}$ http://www.astro.uni-koeln.de/cdms

5 http://spec.jpl.nasa.gov/ 


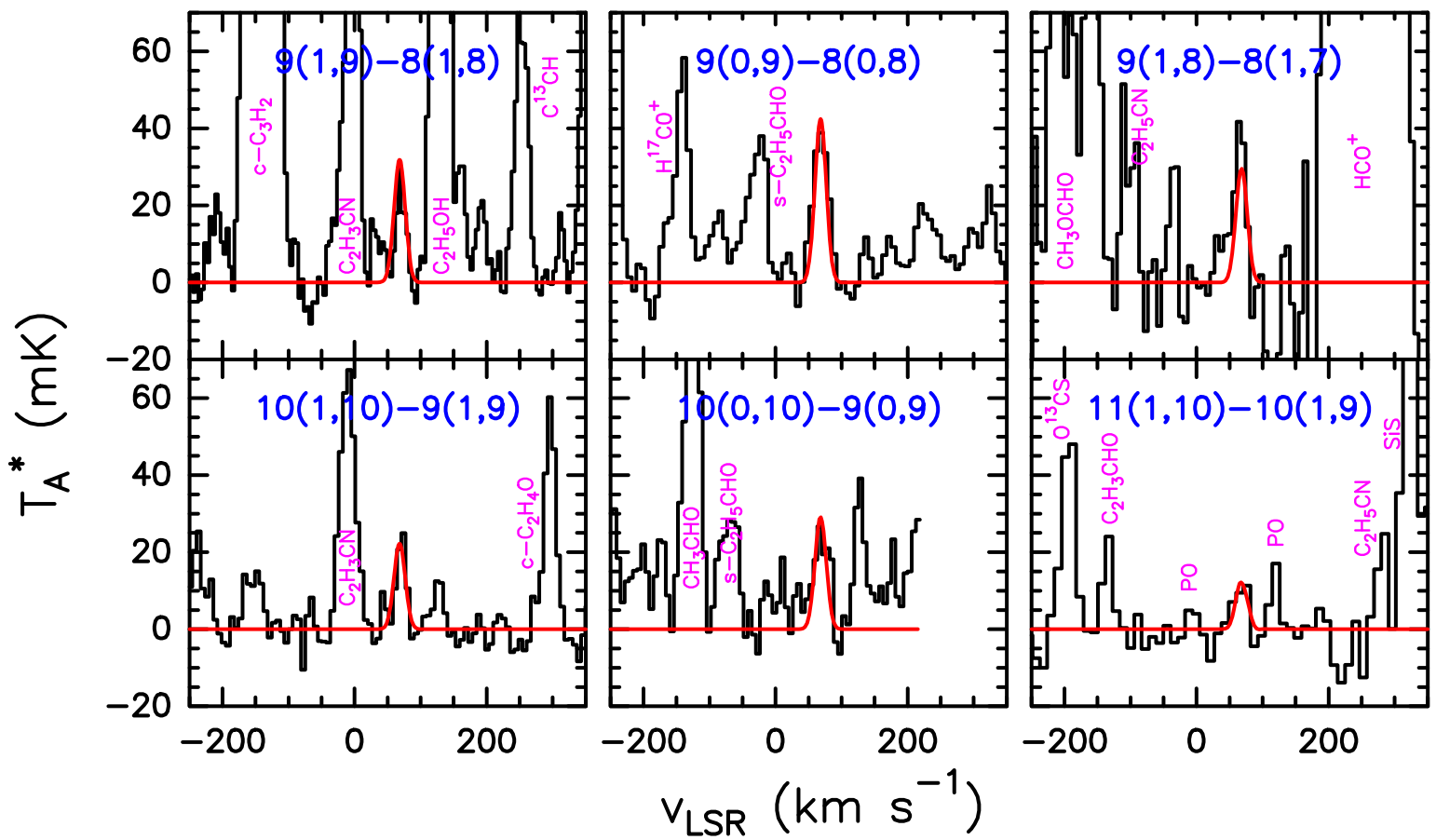

Figure 1. IRAM 30m spectra of Z-HNCHCN towards the Galactic Center quiescent giant molecular cloud G+0.693. The red curves correspond to the LTE best fit obtained with MADCUBA-AUTOFIT. The quantum numbers of each transition are shown in blue in each panel (see also Table 1). Other molecular species identified in the spectra are indicated with magenta labels.

species, while the other four are blended with other species. We checked that the unblended transitions are not associated with any of the $>90$ species we have identified toward this source (Requena-Torres et al. 2008; Rivilla et al. 2018; Zeng et al. 2018; see Figure 1). We note that not a single transition of Z-HNCHCN predicted by the LTE spectrum is missing in the data. The spectroscopic information of the six unblended transitions are summarized in Table 1 , and the spectra are shown in Figure 1. This is the first detection of this species in the ISM.

Then, we used the MADCUBA-AUTOFIT tool that compares the observed spectra with the LTE synthetic spectra, taking into account all the transitions considered, and it provides the best non-linear least-squared fit using the Levenberg-Marquardt algorithm. The free parameters in the fit are: column density $(N)$ of the molecule, excitation temperature $\left(T_{\mathrm{ex}}\right)$, velocity $(v)$, and full width half maximum $(F W H M)$. We did not apply a beam dilution factor since it is well known that the molecular emission towards this source is extended over the beam (e.g. Requena-Torres et al. 2006; Martín et al. 2008; Rivilla et al. 2018). For Z-HNCHCN, since the algorithm did not converge leaving all four parameters free, we fixed the linewidth to $20 \mathrm{~km} \mathrm{~s}^{-1}$, which reproduces well the observed spectra and it is consistent with the values inferred for other -CN species (Zeng et al. 2018), and rerun AUTOFIT. The results of the fit are summarized in Table 2. We derived an excitation temperature of $8 \pm 2 \mathrm{~K}$, very similar to that determined for other complex species in this region (Requena-Torres et al. 2008; Zeng et al. 2018), and a column density of $(2.0 \pm 0.6) \times 10^{14} \mathrm{~cm}^{-2}$. We present in Table 1 the velocity integrated intensity $\left(\int T_{\mathrm{A}}^{*} d \mathrm{v}\right)$ of the identified transitions resulting from the fit. We calculated the detection level of each transition comparing the velocity integrated intensity with $r m s \times \sqrt{\delta \mathrm{v} / F W H M} \times F W H M$, where $r m s$ is the noise measured in line-free spectral ranges close to each transition, and $\delta \mathrm{v}$ is the spectral resolution of the spectra. Three of the identified transitions of Z-HNCHCN are above $13 \sigma$, two above $7 \sigma$ and one above $4 \sigma$ (Table 1 ).

We repeated the analysis for the E-isomer. We identified eight transitions above $>4 \sigma$, of which five are unblended (Table 1 and Figure 2). Since the AUTOFIT algorithm did not converge leaving $T_{\mathrm{ex}}$ as a free parameter, we fixed it to the value found for the Z-isomer, $8 \mathrm{~K}$. We obtained a column density of $(0.33 \pm 0.03) \times 10^{14} \mathrm{~cm}^{-2}$. Three transitions are detected above $11 \sigma$, and two above $6 \sigma$ (Table 1 ). Both conformers have velocities of around $\sim 68 \mathrm{~km} \mathrm{~s}^{-1}$, consistently with many other molecules observed towards this region (see e.g. Zeng et al. 2018). The molecular ratio between the two conformers is $[\mathrm{Z} / \mathrm{E}]=6.1 \pm 2.4$. The total molecular abundance of $\mathrm{C}$-cyanomethanimine, considering both isomers, is $1.74 \times 10^{-9}$.

We derived the fractional molecular abundances by dividing their column densities by the $\mathrm{H}_{2}$ column density $\left(\mathrm{N}_{\mathrm{H}_{2}}\right)$ measured in $\mathrm{G}+0.693$. We adopted $\mathrm{N}_{\mathrm{H}_{2}}=1.35 \times 10^{23}$ $\mathrm{cm}^{-2}$ as inferred by Martín et al. (2008) from $\mathrm{C}^{18} \mathrm{O}$ observations. In our calculations, we assumed that all molecules show a similar spatial distribution than $\mathrm{C}^{18} \mathrm{O}$, i.e. all molecules arise from the same volume. The derived abundances are presented in Table 2 . The Z-isomer has a relatively high abundance of $1.5 \times 10^{-9}$, which is comparable to those of other nitrogen-bearing species in this source such as $\mathrm{CH}_{3} \mathrm{CN}$ or $\mathrm{HC}_{5} \mathrm{~N}$ and higher than those of e.g. $\mathrm{CH}_{3} \mathrm{NCO}$ and $\mathrm{C}_{2} \mathrm{H}_{5} \mathrm{CN}$ (Zeng et al. 2018).

We also searched in the spectra of $\mathrm{G}+0.693$ for other molecules that have been proposed as possible precursors of HNCHCN (see further discussion in Section 4): 
the cyanogen radical $(\mathrm{CN})$, methanimine $\left(\mathrm{CH}_{2} \mathrm{NH}\right)$ and cyanogen (NCCN). Since CN is optically thick towards $\mathrm{G}+0.693$, we have analyzed the optically thin isotopologue ${ }^{13} \mathrm{CN}$. The spectra and the spectroscopic information of the studied ${ }^{13} \mathrm{CN}$ transitions are shown in Appendix A. The results of AUTOFIT are presented in Table 2. Assuming the isotopic ratio of ${ }^{12} \mathrm{C} /{ }^{13} \mathrm{C} \sim 21$ derived in this source by Armijos-Abendaño et al. (2014), we obtained a CN fractional abundance of $1.5 \times 10^{-8}$. The results of $\mathrm{CH}_{2} \mathrm{NH}$ were previously presented in Zeng et al. (2018) and are also shown in Table 2.

Since the detection of NCCN is not possible through radio and millimeter observations due to the lack of a permanent electric dipole moment, we searched for its protonated form, $\mathrm{NCCNH}^{+}$. We confirmed the presence of this species through the detection of the $\mathrm{J}=10-9$ and $\mathrm{J}=11-10$ rotational transitions (see Appendix A). To our knowledge this is the third detection of this species in the ISM after those in the dark cloud TMC-1 and the L483 dense core (Agúndez et al. 2015). Our analysis yielded a fractional molecular abundance of $1.4 \times 10^{-12}$ for this species (Table 2). If we assume a $\left[\mathrm{NCCNH}^{+}\right] /[\mathrm{NCCN}]$ ratio of $\sim 10^{-4}$ as inferred from the chemical modelling of Agúndez et al. (2015), the abundance of NCCN would be $1.4 \times 10^{-8} \mathrm{~cm}^{-2}$.

\section{DISCUSSION}

Due to the lack of detections, very little is known about the formation of HNCHCN. There is no chemical formation route of this species included in the astrochemical databases KIDA $^{6}$ (Wakelam et al. 2012) and UMIST $^{7}$ (McElroy et al. 2013). Zaleski et al. (2013) suggested that radical chemistry on the surface of dust grains might form HNCHCN. More recently, two possible formation routes have been proposed. Vazart et al. (2015) studied the neutral-neutral gas-phase reaction between the cyanogen radical and methanimine:

\section{$\mathrm{CN}+\mathrm{CH}_{2} \mathrm{NH} \rightarrow \mathrm{HNCHCN}+\mathrm{H}$}

A different chemical pathway has been proposed by Shivani et al. (2017) both on the surface of icy dust grains and in the gas phase:

\section{$\mathrm{NCCN}+\mathrm{H}+\mathrm{H} \rightarrow \mathrm{HNCHCN}$}

All proposed formation paths seem to be barrierless, which suggests that both gas-phase and grain surface reactions are able to form efficiently $\mathrm{HNCHCN}$ provided that the precursors are sufficiently abundant. Our data indicate that the reactants of the proposed reactions $\left(\mathrm{CN}, \mathrm{CH}_{2} \mathrm{NH}\right.$ and NCCN) are relatively abundant in $\mathrm{G}+0.693$, with abundances ranging from $4 \times 10^{-9}$ to $1.5 \times 10^{-8}$ (Table 2), which are higher than the derived abundance of $\mathrm{HNCHCN}$ by factor of around 9,2 and 8, respectively. This suggests that these mechanisms might be able to explain the high abundance of HNCHCN $\left(1.74 \times 10^{-9}\right)$ inferred in this cloud.

Since we have detected for the first time both isomers,

6 Kinetic Database for Astrochemistry: http://kida.obs.ubordeaux1.fr

7 http://udfa.ajmarkwick.net/index.php we can use the $[\mathrm{Z} / \mathrm{E}]$ ratio to constrain the proposed formation scenarios. Vazart et al. (2015) showed that the gasphase formation route from $\mathrm{CN}$ and $\mathrm{CH}_{2} \mathrm{NH}$ produces a ratio $[\mathrm{Z} / \mathrm{E}] \sim 1.5$, regardless of the temperature. The calculations by Shivani et al. (2017) predict a [Z/E] ratio of 0.9 in gas-phase and of 1 on the surface of dust grains. Therefore, both pathways fail to explain the observed ratio of $\sim 6$, which might indicate that we are missing key formation routes and/or destruction reactions. A complete study including all the formation and destruction channels of the involved species is needed before drawing firm conclusions.

Interestingly, the $[\mathrm{Z} / \mathrm{E}]$ ratio found in $\mathrm{G}+0.693$ seems to indicate that the two isomers are close to thermodynamic equilibrium at the kinetic temperature $T_{\mathrm{k}}$ of the cloud. If this is the case, the abundances of the isomers are related through the expression:

$$
[Z / E]=\frac{N(Z)}{N(E)}=\frac{1}{g} \times \exp \left(\frac{\Delta E}{T_{\mathrm{k}}}\right)
$$

where $\Delta E$ is the energy difference between the isomers, and $g$ accounts for the statistical weights, which in this case is 1. Takano et al. (1990) derived experimentally an energy difference of $237-382 \mathrm{~K}$, which is in good agreement with the value of $370 \mathrm{~K}$ inferred with the quantum chemical calculations by Zaleski et al. (2013), and with the value of 307 K more recently estimated by Puzzarini (2015). Then, the observed [Z/E] ratio of 6.1 implies a $T_{\mathrm{k}}$ in the range 130-210 $\mathrm{K}$, which is in good agreement with the kinetic temperature found by Zeng et al. (2018) in G+0.693. This suggests that the two isomers are in thermodynamic equilibrium at the $T_{k}$ of the gas. We note that also the populations of other isomers in the ISM seem to be in thermodynamic equilibrium at $\mathrm{T}_{\mathrm{k}}$, as e.g. the conformers of ethyl formate $\left(\mathrm{C}_{2} \mathrm{H}_{5} \mathrm{OCHO}\right)$ in the hot molecular cores located in the W51 and Orion KL regions (Rivilla et al. 2017b; Tercero et al. 2013). Since the isomerization barrier between the E- and Z-isomers of HNCHCN is very high $(15.95 \mathrm{kK}$; Zaleski et al. 2013) this process cannot occur in the ISM. This means that the $T_{\mathrm{k}}$ derived from eq. 3 reflects the temperature at which the molecules were formed. Since the dust in G+0.693 is cold ( $\leq 30 \mathrm{~K}$; Rodríguez-Fernández et al. 2004 ), and the gas temperatures are high $(\sim 50 \mathrm{~K}$ to $\sim 150 \mathrm{~K}$; (e.g. Guesten et al. 1985; Hüettemeister et al. 1993; Rodríguez-Fernández et al. 2001; Ginsburg et al. 2016; Krieger et al. 2017; Zeng et al. 2018), this opens two possible chemical pathways:

i) gas-phase reactions occurring at the high kinetic temperatures of the cloud; and

ii) formation on dust triggered by non-thermal energetic events like cosmic-ray impacts, and their subsequent release by grain sputtering in moderate-velocity shock waves. The latter scenario is plausible in the case of $\mathrm{G}+0.693$ since large-scale low-velocity shocks are widespread in the region due to the encounter of two streams of molecular gas (Hasegawa et al. 1994; Henshaw et al. 2016). However, the current observations do not allow to discriminate between these two possible chemical routes.

Whatever the formation mechanism, our analysis of the first detection in the ISM of the Z-isomer of HNCHCN reveals that its abundance is higher than that of the E-conformer by a factor of 6 . Given the proposed role of $\mathrm{HNCHCN}$ as precursor of adenine (Eschenmoser 


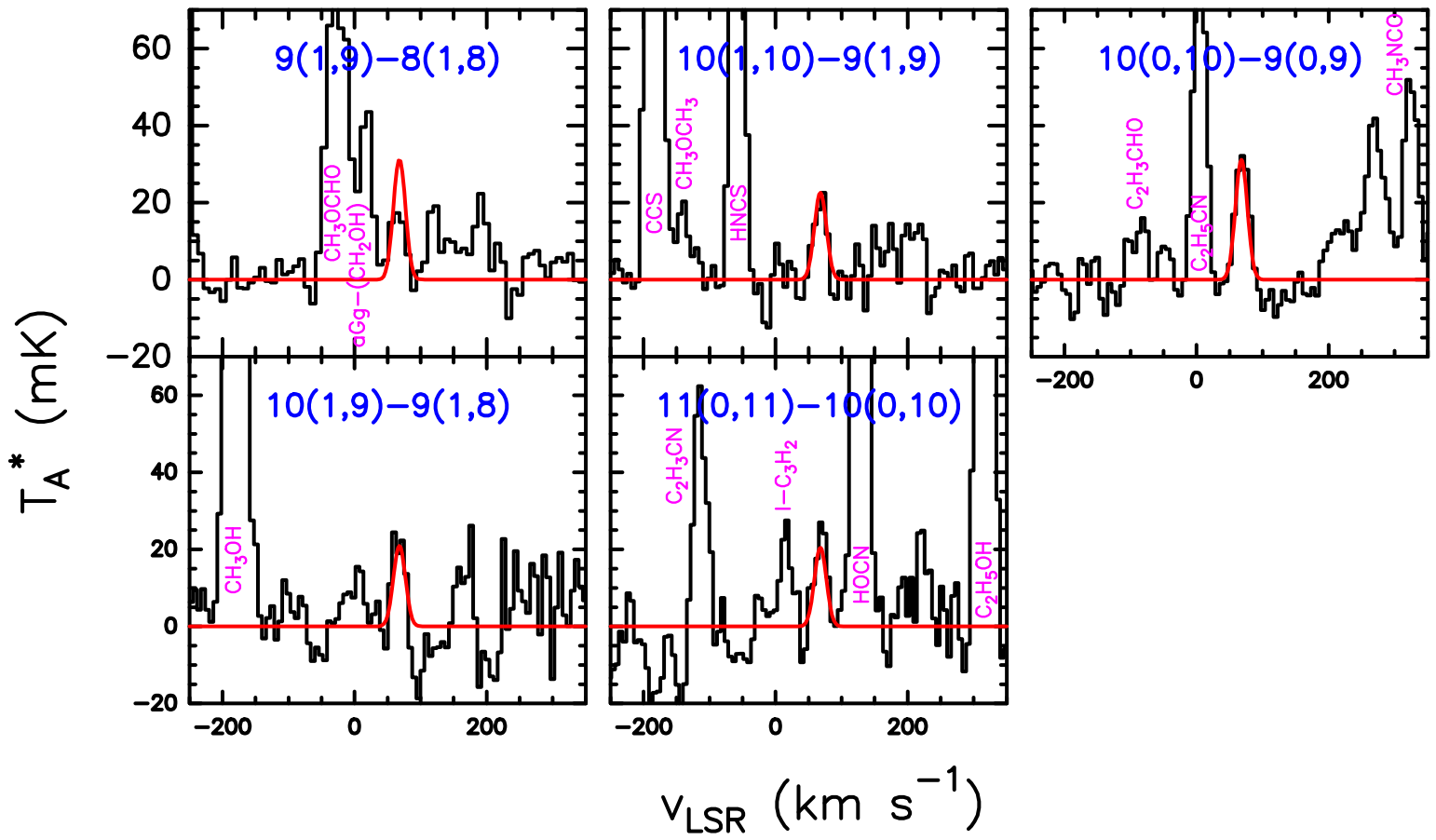

Figure 2. IRAM 30m spectra of E-HNCHCN towards the Galactic Center quiescent giant molecular cloud G+0.693. The red curves correspond to the LTE best fit obtained with MADCUBA-AUTOFIT. The quantum numbers of each transition are shown in blue in each panel (see also Table 1). Other molecular species identified in the spectra are indicated with magenta labels.

Table 2. Derived parameters of the HNCHCN isomers detected towards $\mathrm{G}+0.693$

\begin{tabular}{lccccc}
\hline Species & $\begin{array}{c}\mathrm{N} \\
\left(\times 10^{14} \mathrm{~cm}^{-2}\right)\end{array}$ & $\begin{array}{c}\mathrm{T}_{\mathrm{ex}} \\
(\mathrm{K})\end{array}$ & $\begin{array}{c}\mathrm{v}_{\mathrm{LSR}} \\
\left(\mathrm{km} \mathrm{s}^{-1}\right)\end{array}$ & $\begin{array}{c}\text { FWHM } \\
\left(\mathrm{km} \mathrm{s}^{-1}\right)\end{array}$ & $\begin{array}{c}\text { Abundance } \\
\left(\times 10^{-10}\right)\end{array}$ \\
\hline $\mathrm{Z}-\mathrm{HNCHCN}$ & $2.0 \pm 0.6$ & $8 \pm 2$ & $68.3 \pm 0.8$ & $20^{(a)}$ & 15 \\
$\mathrm{E}-\mathrm{HNCHCN}$ & $0.33 \pm 0.03$ & $8^{(a)}$ & $68.0 \pm 0.8$ & $21 \pm 2$ & 2.4 \\
\hline${ }^{13} \mathrm{CN}$ & $0.94 \pm 0.03$ & $10^{(a)}$ & $71.6 \pm 0.4$ & $18.8 \pm 0.9$ & 7.0 \\
$\mathrm{CN}$ & & & & & $150^{(c)}$ \\
$\mathrm{CH}_{2} \mathrm{NH}^{(b)}$ & $5.4 \pm 0.3$ & $9.7 \pm 0.4$ & $69 \pm 1$ & $25 \pm 1$ & 40 \\
$\mathrm{NCCNH}^{+}$ & $0.0019 \pm 0.004$ & $10^{(a)}$ & $69 \pm 2$ & $22 \pm 5$ & 0.014 \\
$\mathrm{NCCN}$ & & & & & $140^{(d)}$ \\
\hline
\end{tabular}

(a) Parameter fixed in the MADCUBA-AUTOFIT analysis. (b) From Zeng et al. (2018). (c) Assuming the isotopic ratio of ${ }^{12} \mathrm{C} /{ }^{13} \mathrm{C} \sim 21$ derived in $\mathrm{G}+0.693$ by Armijos-Abendaño et al. (2014). (d) Assuming a $\left[\mathrm{NCCNH}^{+}\right] /[\mathrm{NCCN}]$ ratio of $\sim 10^{-4}$, as inferred from chemical modelling by Agúndez et al. (2015).

2007; Chakrabarti \& Chakrabarti 2000; Balucani 2012; Jung \& Choe 2013), the relative high abundance of this species, $1.5 \times 10^{-9}$, argues in favor of an efficient synthesis of key precursors of adenine in space. This is a crucial step to understand how the basic ingredients of life could have been assembled in the ISM before their incorporation to the primitive Earth. The role of $\mathrm{HNCHCN}$ in the formation of more complex nitrile dimers, and in particular adenine, should be addressed in detail with new detections of HNCHCN in more interstellar sources and with chemical modelling.

\section{ACKNOWLEDGEMENTS}

We thank the anonymous referee for his/her instructive comments and suggestions. V.M.R. has received funding from the European Union's H2020 research and innovation programme under the Marie Skłodowska-Curie grant agreement No 664931. J.M.-P. acknowledges partial support by the MINECO and FEDER funding under grants ESP201565597-C4-1 and ESP2017-86582-C4-1-R.

\section{REFERENCES}

Agúndez M., et al., 2015, Astronomy and Astrophysics, 579, L10 Armijos-Abendaño J., Martín-Pintado J., RequenaTorres M. A., Martín S., Rodríguez-Franco A., 2014, Monthly Notices of the Royal Astronomical Society, 446, 3842

Balucani N., 2009, International Journal of Molecular Sciences, 10,2304

Balucani N., 2012, Chemical Society Reviews, 41, 5473

Bernstein M. P., Ashbourn S. F. M., Sandford S. A., Allamandola L. J., 2004, The Astrophysical Journal, 601, 365

Chakrabarti S., Chakrabarti S. K., 2000, Astronomy and Astrophysics, 354, L6

Clemmons J. H., Jasien P. G., Dykstra C. E., 1983, Molecular Physics, 48, 631

Ehrenfreund P., Glavin D. P., Botta O., Cooper G., Bada J. L., 2001, Proceedings of the National Academy of Sciences, 98, 2138

Endres C. P., Schlemmer S., Schilke P., Stutzki J., MÃijller H. S. P., 2016, Journal of Molecular Spectroscopy, 327, 95

Eschenmoser A., 2007, Tetrahedron, 63, 12821

Ginsburg A., et al., 2016, Astronomy \& Astrophysics, 586, A50

Guesten R., Walmsley C. M., Ungerechts H., Churchwell E., 1985, Astronomy and Astrophysics, 142, 381 
Hasegawa T., Sato F., Whiteoak J. B., Miyawaki R., 1994, The Astrophysical Journal Letters, 429, L77

Henshaw J. D., Longmore S. N., Kruijssen J. M. D., 2016, Monthly Notices of the Royal Astronomical Society: Letters, 463, L122

Hüettemeister S., Wilson T. L., Bania T. M., Martín-Pintado J., 1993, Astronomy and Astrophysics, 280, 255

Jung S. H., Choe J. C., 2013, Astrobiology, 13, 465

Kaiser R. I., Balucani N., 2001, Accounts of Chemical Research, 34,699

Krieger N., et al., 2017, The Astrophysical Journal, 850, 77

Martín S., Requena-Torres M. A., Martín-Pintado J., Mauersberger R., 2008, The Astrophysical Journal, 678, 245

McElroy D., Walsh C., Markwick A. J., Cordiner M. A., Smith K., Millar T. J., 2013, Astronomy and Astrophysics, 550, A36

Melosso M., et al., 2018, Astronomy and Astrophysics, 609, A121

Müller H. S. P., Thorwirth S., Roth D. A., Winnewisser G., 2001, Astronomy and Astrophysics, 370, L49

Müller H. S. P., Schlöder F., Stutzki J., Winnewisser G., 2005, Journal of Molecular Structure, 742, 215

Oró J., 1961, Nature, 191, 1193

Pickett H. M., Poynter R. L., Cohen E. A., Delitsky M. L., Pearson J. C., Müller H. S. P., 1998, Journal of Quantitative Spectroscopy and Radiative Transfer, 60,883

Puzzarini C., 2015, The Journal of Physical Chemistry A, 119, 11614

Requena-Torres M. A., Martín-Pintado J., Rodríguez-Franco A., Martín S., Rodríguez-Fernández N. J., de Vicente P., 2006, Astronomy \& Astrophysics, 455, 971

Requena-Torres M. A., Martín-Pintado J., Martín S., Morris M. R., 2008, The Astrophysical Journal, 672, 352

Rivilla V. M., Fontani F., Beltrán M. T., Vasyunin A., Caselli P., Martín-Pintado J., Cesaroni R., 2016, The Astrophysical Journal, 826, 161

Rivilla V. M., Beltrán M. T., Cesaroni R., Fontani F., Codella C., Zhang Q., 2017a, Astronomy and Astrophysics, 598, A59

Rivilla V. M., Beltrán M. T., Martín-Pintado J., Fontani F., Caselli P., Cesaroni R., 2017b, Astronomy and Astrophysics, 599, A26

Rivilla V. M., et al., 2018, Monthly Notices of the Royal Astronomical Society,

Rodríguez-Fernández N. J., Martín-Pintado J., de Vicente P., Fuente A., Hüttemeister S., Wilson T. L., Kunze D., 2000, Astronomy and Astrophysics, 356, 695

Rodríguez-Fernández N. J., Martín-Pintado J., Fuente A., de Vicente P., Wilson T. L., Hüttemeister S., 2001, Astronomy and Astrophysics, 365, 174

Rodríguez-Fernández N. J., Martín-Pintado J., Fuente A., Wilson T. L., 2004, Astronomy and Astrophysics, 427, 217

Shivani Misra A., Tandon P., 2017,

Research in Astronomy and Astrophysics, 17, 1
Smith I. W. M., Talbi D., Herbst E., 2001, Astronomy and Astrophysics, 369, 611

Takano S., Sugie M., Sugawara K.-i., Takeo H., Matsumura C., Masuda A., Kuchitsu K., 1990, Journal of Molecular Spectroscopy, 141, 13

Tercero B., Kleiner I., Cernicharo J., Nguyen H. V. L., López A., Mun̄oz Caro G. M., 2013, The Astrophysical Journal Letters, $770, \mathrm{~L} 13$

Vazart F., Latouche C., Skouteris D., Balucani N., Barone V., 2015, The Astrophysical Journal, 810, 111

Wakelam V., et al., 2012, The Astrophysical Journal Supplement, 199, 21

Yim M. K., Choe J. C., 2012, Chemical Physics Letters, 538, 24

Zaleski D. P., et al., 2013, The Astrophysical Journal Letters, 765, L10

Zeng S., et al., 2018, Monthly Notices of the Royal Astronomical Society, 
APPENDIX A: SPECTRA OF ${ }^{13} \mathrm{CN}$ AND $\mathrm{NCCNH}^{+}$
This paper has been typeset from a $\mathrm{T}_{\mathrm{E}} \mathrm{X} / \mathrm{LAT} \mathrm{T} \mathrm{X}$ file prepared by the author. 
Table A1. Spectroscopic information from CDMS molecular database of the ransitions of ${ }^{13} \mathrm{CN}$ detected towards $\mathrm{G}+0.693$.

\begin{tabular}{|c|c|c|c|}
\hline $\begin{array}{c}\text { Frequency } \\
\quad(\mathrm{GHz})\end{array}$ & Transition & $\begin{array}{c}\log A_{\mathrm{ul}} \\
\left(\mathrm{s}^{-1}\right)\end{array}$ & $\begin{array}{l}\mathrm{E}_{\mathrm{up}} \\
(\mathrm{K})\end{array}$ \\
\hline 108.631121 & $1110-0101$ & -5.0188 & 5.2 \\
\hline 108.636923 & $1111-0101$ & -5.0172 & 5.2 \\
\hline 108.638212 & $1211-0110$ & -5.4444 & 5.2 \\
\hline 108.643590 & $1212-0111$ & -5.5926 & 5.2 \\
\hline 108.644346 & $1210-0111$ & -5.0188 & 5.2 \\
\hline 108.645064 & $1211-0111$ & -5.5620 & 5.2 \\
\hline 108.651297 & $11112-0101$ & -5.0095 & 5.2 \\
\hline 108.657646 & $1212-0112$ & -5.1411 & 5.2 \\
\hline 108.658948 & $1211-0112$ & -5.4775 & 5.2 \\
\hline 108.780010 & $1223-0112$ & -4.9788 & 5.2 \\
\hline 108.782374 & $1222-0111$ & -5.1107 & 5.2 \\
\hline 108.786982 & $1221-0110$ & -5.2430 & 5.2 \\
\hline 108.793753 & $1221-0111$ & -5.3502 & 5.2 \\
\hline 108.796400 & $1222-0112$ & -5.5604 & 5.2 \\
\hline 108.807788 & $1221-0112$ & -6.4897 & 5.2 \\
\hline 108.986836 & $1101-0101$ & -7.2578 & 5.2 \\
\hline 109.217567 & $1212-0101$ & -6.1603 & 5.2 \\
\hline 109.218323 & $1210-0101$ & -6.0475 & 5.2 \\
\hline 109.218919 & $1211-0101$ & -6.0932 & 5.2 \\
\hline
\end{tabular}

Table A2. Spectroscopic information from CDMS molecular database of the transitions of $\mathrm{NCCNH}^{+}$

\begin{tabular}{ccccc}
\cline { 2 - 5 } detected towards G+0.693. & $\begin{array}{c}\text { Frequency } \\
(\mathrm{GHz})\end{array}$ & Transition & $\begin{array}{c}\log \mathrm{A}_{\mathrm{ul}} \\
\left(\mathrm{s}^{-1}\right)\end{array}$ & $\begin{array}{c}\mathrm{E}_{\mathrm{up}} \\
(\mathrm{K})\end{array}$ \\
\cline { 2 - 5 } & 88.758103 & $10-9$ & -3.7928 & 23 \\
& 97.633424 & $11-10$ & -3.6668 & 28 \\
\hline
\end{tabular}

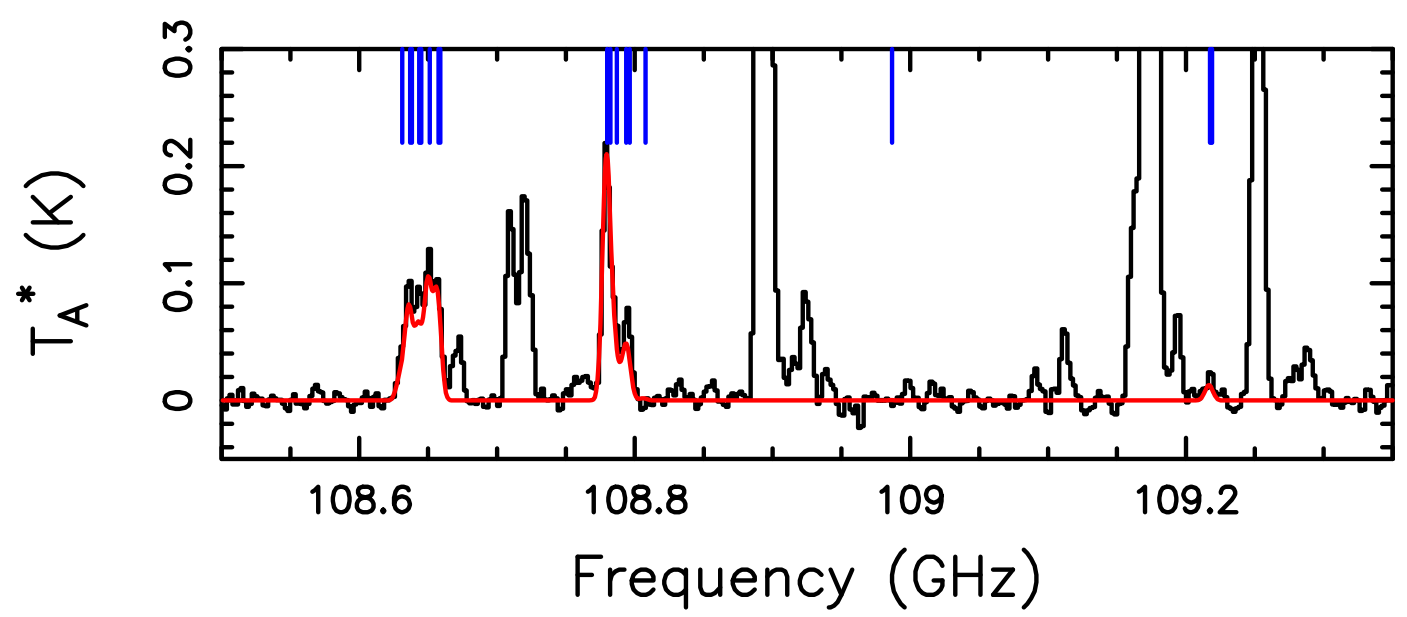

Figure A1. IRAM 30m spectra of ${ }^{13} \mathrm{CN}$ towards the Galactic Center quiescent giant molecular cloud $\mathrm{G}+0.693$. The red curves correspond to the LTE best fit obtained with MADCUBA-AUTOFIT. The vertical blue lines indicate the position of the transitions presented in Table A1. 


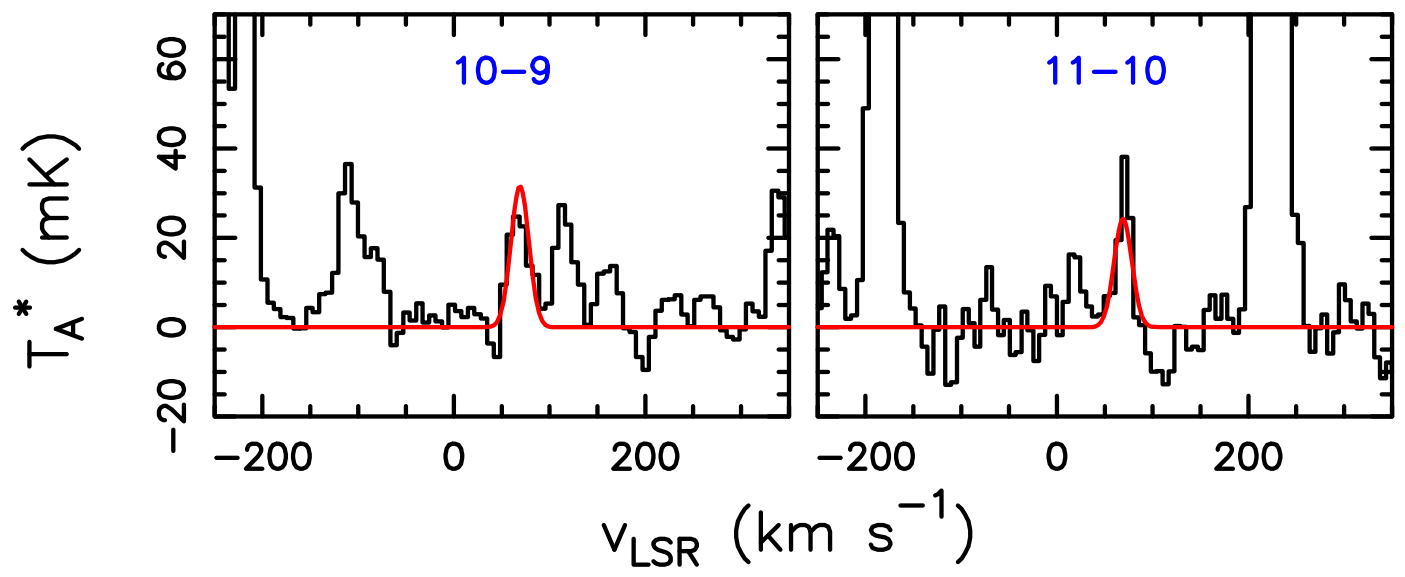

Figure A2. IRAM 30m spectra of $\mathrm{NCCNH}^{+}$towards the Galactic Center quiescent giant molecular cloud G+0.693. The red curves correspond to the LTE best fit obtained with MADCUBA-AUTOFIT. 